\title{
Bioinformatics and Regression Analyses Manifest Tumor-Specific miRNA Expression Dynamics in Pediatric Embryonal Malignancies
}

\author{
Maria Braoudaki, Dimitrios-Dionysios Koutsouris, Member, IEEE, Ioannis Kouris, Anna Paidi, \\ Georgia Koutsouri, George I. Lambrou
}

\begin{abstract}
Pediatric Central Nervous System (CNS) neoplasms are the second most prevalent tumors of childhood. Further on, prognosis of this type of neoplasms still remain poor and the comprehension of the etiology and pathogenesis of the disease still remains scarce. Several reports have identified microRNAs as significant molecules in the development of central nervous system tumors and propose that they might compose key molecules underlying oncogenesis. In a previous study we have identified several miRNAs, common to different subtypes of pediatric embryonal CNS malignancies as well as, we have identified miRNAs that manifest significant dynamics with respect to their expression and the neoplasmatic subtype. Overall, 19 tumor cases from children diagnosed with embryonal brain tumors were investigated. As controls, children who suffered a sudden death underwent autopsy and were not present with any brain malignancy were used (13 samples of varying localization). Our experimental approach included microarrays covering 1211 miRNAs, which appeared to manifest tumor-specific dynamics. In conclusion, it appeared that certain miRNAs are neoplasm specific and in particular, their expression manifests linear dynamics. Thus, the investigation of miRNA expression in pediatric embryonal brain tumors might contribute towards the discovery of tumor-specific miRNA signatures, which could potentially afford the identification of gene-specific biomarkers related to diagnosis, prognosis and patient targeted therapy, as well as help us understand oncogenetic dynamics.
\end{abstract}

\section{INTRODUCTION}

Central nervous system (CNS) neoplasms of childhood of embryonal origin comprise of a group of malignant neoplasms that are by far the largest group of malignant brain tumors in childhood, highly associated with increased mortality and long-term morbidity. All embryonal neoplasms are staged as grade IV malignancies and they consist of medulloblastomas (MBs) and atypical teratoid/rhabdoid tumors (AT/RTs), among others [1].

MB is with the Department of Life and Environmental Sciences, School of Life and Health Sciences, University of Hertfordshire and the National and Kapodistrian University of Athens, First Department of Pediatrics, Hematology/Oncology Unit (email: mbraoudak@med.uoa.gr). DDK is with the National Technical University of Athens, School of Electrical Engineering, Biomedical Engineering Laboratory (email: dkoustou@biomed.ntua.gr). IK is with the National Technical University of Athens, School of Electrical Engineering, Biomedical Engineering Laboratory (email: ikouris@biomed.ntua.gr). AP is with the National Technical University of Athens, School of Electrical Engineering, Biomedical Engineering Laboratory (email: paidi.anna@gmail.com). GK is
Medulloblastoma is considered the most common malignant brain tumor of childhood, accounting for $20 \%$ of cases [2]. Risk stratification based on clinical parameters is insufficient for accurate prognostication [3]. Current treatment options include surgery, chemotherapy and radiotherapy [4]. However, age limitations for the delivery of radiotherapy have been set due to the vulnerability of the developing brain to radiotherapy-induced neurocognitive deficits [5]. Notwithstanding, aggressive multimodal therapy has improved the prognosis for children with $\mathrm{MB}$, nearly one third of patients will eventually succumbed to progressive tumors.

AT/RT is a highly malignant CNS tumour consisting of 1$2 \%$ of paediatric brain tumours and accounts for at least $10 \%$ of CNS tumours in infants, due to the predominance in children younger of 3 years old. The survival of children younger than 3 years of age remains poor, particularly for patients with supratentorial tumours and those with metastatic disease [2]. It can be supratentorial, especially in cerebral hemispheres, or infratentorial, especially in the cerebellar hemispheres, cerellopontine angle and brain stem mainly in children younger than 2 years of age. Prognosis of $\mathrm{AT} / \mathrm{RT}$ is dismal, while there are no protocols aimed specifically for this type [2].

Undeniably, thus far, expression profile analysis has revealed several miRNA signatures related to malignant pediatric brain tumors [6-11]. In a previous work we have examined the expression signatures of pediatric CNS tumors and in the present report we attempted to identify further expression dynamics in those neoplasms [12].

\section{MATERIALS AND METHODS}

\section{A. Patients and Tumor Samples}

Patient sampling has been reported previously [12]. In brief, in the present study, 19 embryonal tumor samples were

with the National Technical University of Athens, School of Electrical Engineering, Biomedical Engineering Laboratory (email: gkoutsou@biomed.ntua.gr). GIL is with the National Technical University of Athens, School of Electrical Engineering, Biomedical Engineering Laboratory and the National and Kapodistrian University of Athens, First Department of Pediatrics, Hematology/Oncology Unit (corresponding author: Thivon \& Levadeias 8, 15127, Goudi, Athens, Greece, +30 210 7467427; e-mail: glamprou@med.uoa.gr).

The present work was supported in part from the National and Kapodistrian University of Athens, Medical School. 
Accepted Manuscript.

Paper presented at the $201840^{\text {th }}$ Annual International Conference of the IEEE Engineering in Medicine and Biology Society (EMBC), 18/07/2018 - 21/07/2018, Honolulu, HI, USA.

studied from children diagnosed with medulloblastomas (MBs) $(n=15)$ or atypical teratoid/rhabdoid tumors (AT/RT) $(n=4)$ diagnosed according to the $2007 \mathrm{WHO}$ criteria [13]. As controls, 13 samples were used; The First-Choice Human Brain Reference RNA was used (Ambion, Austin, TX, USA) and 12 samples were obtained from deceased children who underwent autopsy and were not present with any brain distortion, including the following anatomic locations: cerebellum $(n=3)$, medulla oblongata $(n=3)$, parietal lobe $(n=3)$ and temporal lobe $(n=3)$. In addition, we have utilized an independent group of Germinoma (GE) tumors. GEs are considered childhood tumors of embryonic origin, arising from primordial germ cells that have migrated aberrantly during embryonic development and subsequently undergone malignant transformation [14]. GEs samples were extracted from the GSE19347 dataset. The study protocol conformed to the ethical guidelines of the 1975 Declaration of Helsinki. The present study was conducted with the approval of "Aghia Sophia" Children's Hospital Ethics Committee (Protocol No. 35/19. 16/09/13).

\section{B. Experimental Procedure, Bioinformatics and Regression Analyses}

Experimental procedures have been previously described in detail [12]. The total gene signals were extracted using the Imagene 6.0 software (Biodiscovery Inc., USA). Data were pre-processed and sorted with Microsoft Excel ${ }^{\circledR}$. Probe replicates were combined and the mean was calculated, which was further used for analysis. Filtering was performed based on the signal intensity. Background correction was performed by subtracting the median local background from the median signal intensity as previously reported [15]. Normalization was performed using the quantile normalization algorithm. MicroRNAs were considered to be significantly differentially expressed (DE) if they obtained a p-value $<0.05$ and an $\mathrm{FDR} \leq 0.05$. The multiparameter analyses were performed with the MATLAB ${ }^{\circledR}$ simulation environment (The Mathworks, Inc, Natick, MA). The two tailed student t-test was used to test the mean differences between two groups. Regression was performed using the $y=a x+b$ and $y=a x^{b}$ functions. Venn diagrams were generated with the Venn Diagram Calculator from the Bioinformatics, Evolutionary Genomics Group of the University of Gent ${ }^{1}$.

\section{RESULTS}

\section{A. Patient Cohort}

Patient descriptive statistics have been reported previously [12]. In brief, the patient cohort included 8 males and 11 females, with minimum age of 0.03 years to maximum 10.2 years (mean age 5.65 years). The median age of MB patients was 7.5 years with a male/female ratio of 1:1.The median age for the AT/RT patients was 1.1 years with a male/female ratio of 1:3, and the median age of the non-malignant cohort was 9 years with a male/female ratio of 1:0.

\section{B. Venn Diagrams Between MB and AT/RT Tumors}

In our previous study, 113 DEs miRNAs were identified (with a significance level $p<0.05$ and $F D R<0.05$ ) between embryonal tumors (MBs and AT/RTs) as compared to the control group. Overall, a total of 54 miRNAs $(47.8 \%)$ were

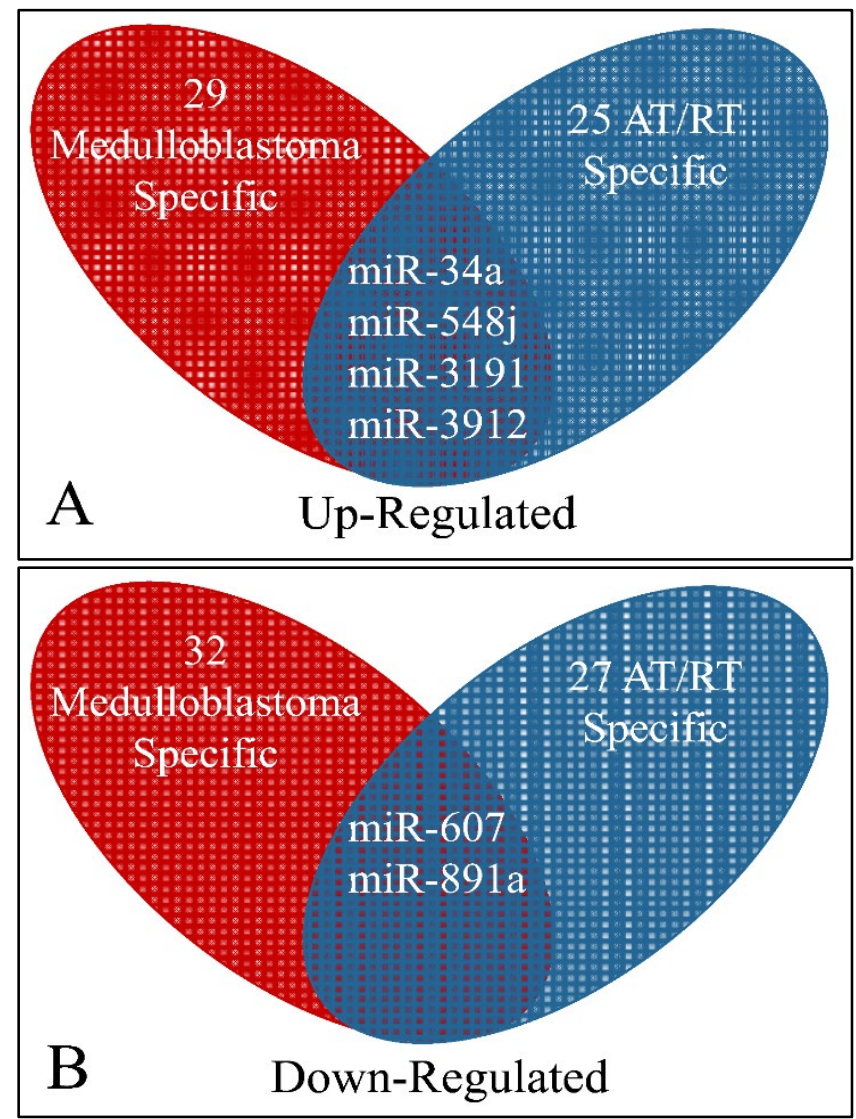

over-expressed and 59 miRNAs (52.2\%) were downregulated. Among them, 107 miRNAs were characterized as tissue-specific. Venn diagram analysis manifested six miRNAs; miR-34a, miR-548j, miR-607, miR-891a, miR3191 and miR-3912. Those miRNAs were found to be commonly differentially expressed in both medulloblastomas as well as in teratoid tumors. Specifically, miR-34a, miR-548j, miR-3191 and miR-3912 were overexpressed, whereas miR-607 and miR-891a were underexpressed. This was found to be true for all tested embryonal tumor samples as compared to the control group. The overlapping relationships of the DE miRNAs among both tumor types is presented in Fig. 1.

Figure 1. Commonly expressed miRNAs among the differentially expressed miRNAs. Venn diagrams illustrate the overlapping relationship of the number of up-regulated miRNAs among MBs and AT/RTs (A) and down-regulated miRNAs among MBs and AT/RTs (B). Overall, 61 miRNAs were specifically differentially expressed in MBs (29 up-regulated 
Accepted Manuscript.

Paper presented at the $201840^{\text {th }}$ Annual International Conference of the IEEE Engineering in Medicine and Biology Society (EMBC), 18/07/2018 - 21/07/2018, Honolulu, HI, USA.

and 32 down-regulated), 52 were specifically differentially expressed in AT/RTs (25 miRNAs up-regulated and 27 down-regulated). Among all of them, 6 were co-differentially expressed in both tumor types (4 co-upregulated and 2 co-down-regulated).

\section{Regression Analysis}

respectively) with a descending pattern from MBs to control samples (Fig. 2B, Fig. 2E). miR-3681 and miR-382 manifested similarly linear dynamics $\left(R^{2}=0.8356\right.$ and $R^{2}=0.9416$ respectively) yet descending from control to
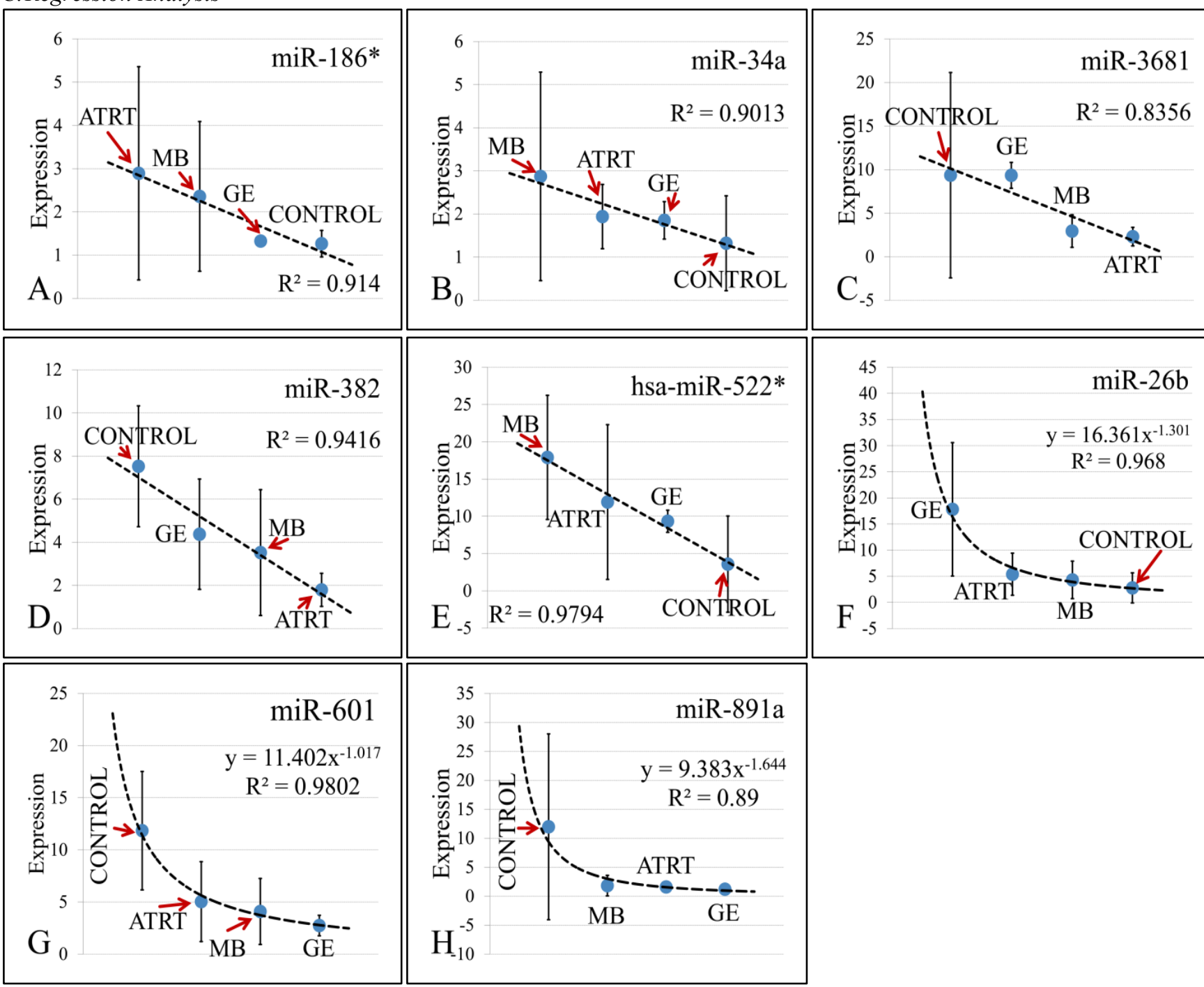

Figure 2. Regressions of the mean expression values of embryonal tumors and controls following meta-analysis. Five miRNAs have manifested linear regression dynamics. In particular, miR-186* $(p=0.049)(\mathbf{A})$, miR-34a (B) $(p=0.043)$, miR-3681 (C) $(p=0.062)$, miR-382 (D) $(p=0.012)$ and miR-522* (E) $(p=0.0013)$ could be linearly simulated obtaining a $R^{2}>0.9$ (except for miR-3681). At the same time miR-26v $(\mathbf{F})(p=0.004), \mathrm{miR}-601(\mathbf{G})(p=0.0022)$ and miR-891a (H) $(p=0.05)$ could be efficiently modelled with functions in the form of $y=a x^{b}$.

Venn diagrams showed that specific miRNAs were similarly up- and down-regulated in MBs and AT/RTs. Based on the previously reported expression levels of commonly differentially expressed miRNAs we have tested those miRNAs for their expression dynamics by regressing their expression values and trying to identify a descending or ascending expression pattern. In particular, miR-186* manifested linear expression dynamics $\left(R^{2}=0.914\right)$ with a descending pattern from AT/RT to control samples (Fig. 2A). miR34a and miR-522* manifested also linear expression dynamics $\left(R^{2}=0.9013\right.$ and $R^{2}=0.9794$
AT/RT samples (Fig. 2C, Fig. 2D). Further on, miR-26b was modelled with an exponential function manifesting excellent dynamics $\left(R^{2}=0.968\right)$ and a descending pattern from GE samples to control samples (Fig. 2F). Finally, miR-601 and miR-891a manifested similar dynamics $\left(R^{2}=0.9802\right.$ and $R^{2}=0.89$ respectively) descending from control to $\mathrm{GE}$ samples (Fig. 2G, 2H).

Further on, in a previous report we have shown that unsupervised hierarchical clustering algorithms were in position to partly distinguish between the different tumors types (data not shown). In particular, it has been found that 
germinomas could be distinctively separated by $\mathrm{MB}$ and AT/RT tumors, while MBs and AT/RTs formed two distinctive groups. For further details the reader is referred to the work of Braoudaki et al. (2014), where the results of unsupervised clustering are presented in detail [12].

\section{DISCUSSION}

In the present work we performed miRNA microarray analysis in pediatric patients with embryonal CNS neoplasms to investigate whether the differential expression of miRNA genes manifests dynamics with respect to tumor type. We have identified several miRNAs that were tumor-specific and at the same time we have identified miRNA that were commonly up- and down-regulated in pediatric embryonal CNS tumors. Our final aim was to identify and provide additional evidence regarding potential miRNA expression signatures relative to their dynamics. To the best of our knowledge, few studies have been comparatively focusing on identifying miRNA biomarkers related to $\mathrm{MB}$ and $\mathrm{AT} / \mathrm{RT}$ diagnostics and therapeutics $[6,10,16,17]$, but also on the possible dynamics that miRNAs could manifest in CNS tumorigenesis. Thus, to our knowledge, this is the first attempt aiding specifically to evaluate an association between miRNA expression pattern dynamics in pediatric MBs and AT/RTs.

By performing miRNA microarrays, 113 miRNAs were found to be differentially expressed, between embryonal tumors and control samples. As aforementioned, 107 were tissue-specific and 6 miRNAs were identified to manifest common expression profile between the two tumor types, yet eight miRNAs manifested linear dynamics. In particular, miR-186* appeared to be linearly regulated, while it was upregulated as compared to control samples. In a previous report it has been found that miR-186* is deleted in medulloblastoma [18], while CRNDE negatively regulates miR-186* and decreases the expression levels of XIAP and PAK7 in gliomas [19]. Similarly, miR-34a manifested similar dynamics. Previous reports set miR034a as a tumor suppressor miRNA in medulloblastoma [20, 21], while another report defines that miR-34a as a chemoresistance factor [22]. Finally, concerning our findings for miR-3681, miR-382, miR-522* and miR-26b, no other reports have mentioned their role in medulloblastoma and atypical teratoid/rhabdoid tumors. The reverse behavior was observed for miR-601 and miR-891a i.e. those miRNAs were downregulated as compared to control samples. No previous reports have been found concerning the role of miR-601 and miR-891a in medulloblastoma and atypical teratoid/rhabdoid tumors.

\section{CONCLUSION}

The current study was shown to provide significant insights into the growing role of several miRNA signatures in pediatric embryonal CNS neoplasms including MBs and AT/RTs. There was good evidence that miRNA signatures could serve as potential markers for tumor subtype and aggression. Further on, the fact that miRNA expression manifested certain dynamics patterns it could imply that they participate in the mechanistic of pediatric CNS tumorigenesis.

\section{ACKNOWLEDGMENT}

Not Applicable.

\section{REFERENCES}

A. Fibriani, R. Wisaksana, A. Indrati, Y. Hartantri, D. van de Vijver, M. Schutten, et al., "Virological failure and drug resistance during first line anti-retroviral treatment in Indonesia," J Med Virol, vol. 85, pp. 1394-401, Aug 2013.

[2] J. A. Heath, S. Zacharoulis, and M. W. Kieran, "Pediatric neurooncology: current status and future directions," Asia Pac J Clin Oncol, vol. 8, pp. 223-31, Sep 2012.

[3] A. Gokhale, R. Kunder, A. Goel, R. Sarin, A. Moiyadi, A. Shenoy, et al., "Distinctive microRNA signature of medulloblastomas associated with the WNT signaling pathway," J Cancer Res Ther, vol. 6, pp. 521-9, Oct-Dec 2010.

[4] L. A. Fernandez, P. A. Northcott, M. D. Taylor, and A. M. Kenney, "Normal and oncogenic roles for microRNAs in the developing brain," Cell Cycle, vol. 8, pp. 4049-54, Dec 152009.

[5] S. Rutkowski, K. von Hoff, A. Emser, I. Zwiener, T. Pietsch, D Figarella-Branger, et al., "Survival and prognostic factors of early childhood medulloblastoma: an international meta-analysis," $J$ Clin Oncol, vol. 28, pp. 4961-8, Nov 202010.

[6] E. Ferretti, E. De Smaele, A. Po, L. Di Marcotullio, E. Tosi, M. S Espinola, et al., "MicroRNA profiling in human medulloblastoma," Int J Cancer, vol. 124, pp. 568-77, Feb 12009.

[7] D. R. Lucon, S. Rocha Cde, R. B. Craveiro, D. Dilloo, I. A Cardinalli, D. P. Cavalcanti, et al., "Downregulation of $14 \mathrm{q} 32$ microRNAs in Primary Human Desmoplastic Medulloblastoma," Front Oncol, vol. 3, p. 254, 2013.

[8] Y. Liu, K. Tang, W. Yan, Y. Wang, G. You, C. Kang, et al., "Identifying Ki-67 specific miRNA-mRNA interactions in malignant astrocytomas," Neurosci Lett, vol. 546, pp. 36-41, Jun 242013

[9] Y. Guan, M. Mizoguchi, K. Yoshimoto, N. Hata, T. Shono, S. O. Suzuki, et al., "MiRNA-196 is upregulated in glioblastoma but not in anaplastic astrocytoma and has prognostic significance," Clin Cancer Res, vol. 16, pp. 4289-97, Aug 152010.

[10] S. Venkataraman, D. K. Birks, I. Balakrishnan, I. Alimova, P. S Harris, P. R. Patel, et al., "MicroRNA 218 acts as a tumor suppressor by targeting multiple cancer phenotype-associated genes in medulloblastoma," J Biol Chem, vol. 288, pp. 1918-28, Jan 182013

[11] M. Visani, D. de Biase, G. Marucci, S. Cerasoli, E. Nigrisoli, M L. Bacchi Reggiani, et al., "Expression of 19 microRNAs in glioblastoma and comparison with other brain neoplasia of grades I-III," Mol Oncol, vol. 8, pp. 417-30, Mar 2014.

[12] M. Braoudaki, G. I. Lambrou, K. Giannikou, V. Milionis, K. Stefanaki, D. K. Birks, et al., "Microrna expression signatures predict patient progression and disease outcome in pediatric embryonal central nervous system neoplasms," J Hematol Oncol, vol. 7, p. 96, Dec 312014.

[13] D. N. Louis, H. Ohgaki, O. D. Wiestler, W. K. Cavenee, P. C. Burger, A. Jouvet, et al., "The 2007 WHO classification of tumours of the central nervous system," Acta Neuropathol, vol. 114, pp. 97-109, Aug 2007.

[14] R. J. Packer, B. H. Cohen, and K. Cooney, "Intracranial germ cell tumors," Oncologist, vol. 5, pp. 312-20, 2000.

[15] E. G. Sifakis, G. I. Lambrou, A. Prentza, S. Vlahopoulos, D. Koutsouris, F. Tzortzatou-Stathopoulou, et al., "Elucidating the identity of resistance mechanisms to prednisolone exposure in acute lymphoblastic leukemia cells through transcriptomic 
Accepted Manuscript.

Paper presented at the $201840^{\text {th }}$ Annual International Conference of the IEEE Engineering in Medicine and Biology Society (EMBC), 18/07/2018 - 21/07/2018, Honolulu, HI, USA.

analysis: A computational approach," J Clin Bioinforma, vol. 1, p. 36, 2011.

[16] D. K. Birks, V. N. Barton, A. M. Donson, M. H. Handler, R. Vibhakar, and N. K. Foreman, "Survey of MicroRNA expression in pediatric brain tumors," Pediatr Blood Cancer, vol. 56, pp. 2116, Feb 2011.

[17] S. T. Sredni, C. C. Huang, F. Bonaldo Mde, and T. Tomita, "MicroRNA expression profiling for molecular classification of pediatric brain tumors," Pediatr Blood Cancer, vol. 57, pp. 1834, Jul 152011.

[18] S. Q. Lv, Y. H. Kim, F. Giulio, T. Shalaby, S. Nobusawa, H. Yang, et al., "Genetic alterations in microRNAs in medulloblastomas," Brain Pathol, vol. 22, pp. 230-9, Mar 2012.

[19] J. Zheng, X. D. Li, P. Wang, X. B. Liu, Y. X. Xue, Y. Hu, et al., "CRNDE affects the malignant biological characteristics of human glioma stem cells by negatively regulating miR-186," Oncotarget, vol. 6, pp. 25339-55, Sep 222015.

[20] Y. N. Fan, D. Meley, B. Pizer, and V. See, "Mir-34a mimics are potential therapeutic agents for p53-mutated and chemo-resistant brain tumour cells," PLoS One, vol. 9, p. e108514, 2014.

[21] T. Thor, A. Kunkele, K. W. Pajtler, A. K. Wefers, H. Stephan, P. Mestdagh, et al., "MiR-34a deficiency accelerates medulloblastoma formation in vivo," Int J Cancer, vol. 136, pp. 2293-303, May 152015.

[22] P. de Antonellis, C. Medaglia, E. Cusanelli, I. Andolfo, L. Liguori, G. De Vita, et al., "MiR-34a targeting of Notch ligand delta-like 1 impairs CD15+/CD133+ tumor-propagating cells and supports neural differentiation in medulloblastoma," PLoS One, vol. 6 , p. e24584, 2011. 